\title{
Electrokinetic Nanoparticle Treatment Success Factors
}

\author{
Henry E. Cardenas, Huayuan Zhong* \\ College of Engineering and Science, Louisiana Tech University, Ruston, LA, USA \\ Email: ^hyzhong89@gmail.com
}

How to cite this paper: Cardenas, H.E. and Zhong, H.Y. (2020) Electrokinetic Nanoparticle Treatment Success Factors. Materials Sciences and Applications, 11, 767-786.

https://doi.org/10.4236/msa.2020.1111052

Received: October 18, 2020

Accepted: November 27, 2020

Published: November 30, 2020

Copyright $\odot 2020$ by author(s) and Scientific Research Publishing Inc. This work is licensed under the Creative Commons Attribution International License (CC BY 4.0).

http://creativecommons.org/licenses/by/4.0/

(c) (i) Open Access

\begin{abstract}
Electrokinetic pozzolanic nanoparticle treatments have been reported in the literature to achieve rapid porosity reductions and deeply penetrating strength enhancement of cement and concrete. The high electric fields required to achieve these results have tended to be accompanied by particle suspension instability. Coagulation is an instability that can limit the efficiency and effectiveness of a treatment by removing particles from suspension. The current study examines how electro-coagulation impacts electrokinetic treatment effectiveness. The nano-pozzolan suspension used in this study was Nalco 1056, alumina-coated silica (24-nm). A threshold electric field strength of $0.4 \mathrm{~V} / \mathrm{cm}$ was identified for avoiding direct electro-coagulation. Treatments conducted at this threshold value exhibited a 50\% strength increase. Treatments conducted above this threshold value exhibited significant particle electro-coagulation losses and strength increases of only $25 \%$. This study found that electro-coagulation was influenced by the electric field strength, through a combination of particle crowding at pore entrances and $\mathrm{pH}$ shifts as driven by electrolysis.
\end{abstract}

\section{Keywords}

Hardening Cement, Electrokinetic Treatment, Nanoparticle, Stability, Suspension, Porosity, Strength

\section{Introduction}

Concrete is one of the more widely used construction materials due to its relatively competitive affordability and versatility. Durability plays a significant role in determining the useful life of a concrete structure. Failures in durability are induced by several factors. High on this list is the problem of elevated capillary porosity [1]. 
Reducing the porosity of an existing concrete structure generally involves surface treatments and structural overlays. In recent years, the application of electrokinetics has been demonstrated to provide deeply penetrating (over $5 \mathrm{~cm}$ ) reductions in porosity. The delivery of colloidal nanoparticles is particularly interesting due to the changes in suspension stability that these materials exhibit. During a given treatment suspended particles are electrophoretically driven well into the structure. At some points, they become unstable and fallout of suspension within the pores [2]. This instability constitutes a significant advantage over the transport of ionic species which can easily leach back out of the structure. In general, the literature has focused on the benefits of these treatments but with little or no examination of particle instability, that can occur prior to pore penetration. This study focused on the question of efficient particle delivery into the capillary pores of hardened cement paste.

\section{Background}

Capillary porosity is an unavoidable characteristic of ordinary Portland cement. It greatly influences durability and strength and is preferably controlled via suitable mix design [3] [4]. These pores are randomly distributed and often exhibit areas of discontinuity, especially in relatively mature concrete. When mix design and batch execution fail to provide the desired result, several approaches have been developed to reduce the surface porosity of hardened concrete structures [5] [6]. In 1992, Lageman reported the use of an electrokinetic approach for porosity reduction by which selected chemicals were transported into concrete capillaries in order to block pore openings [2]. In the past decade, several researchers have demonstrated deeply penetrating and highly effective porosity reductions using electrokinetic treatments applied to cement and concrete [7] [8] [9] [10]. In one study, experimentation, modeling and simulations utilizing alumina-coated-silica nanoparticles achieved electrokinetic porosity reductions of as much as $57 \%$ in hardened cement paste (HCP) [11]. In later work, similar treatments conducted over 6 - 8 weeks of treatment had reduced the porosity of reinforced concrete beams down to less than $3 \%$. The dosages utilized in these studies were relatively high, costly, and exhibited low particle delivery efficiency [12].

The process of electrokinetic nanoparticle transport is governed primarily by electrophoresis. Other transport processes within concrete such as pressure flow, electroosmosis, and diffusion can influence the rate and direction of particle transport [13] [14]. Electrophoretic transport is governed by the applied electric field (E-field), the viscosity and dielectric constant of the fluid, and the Zeta potential of the suspended particle. The Zeta potential is related to the surface charge that surrounds a colloidal particle. A high Zeta potential correlates to a large surface charge that keeps particles well separated (due to high electrostatic repulsion) and thus minimizes particle collisions that cause suspension instability. Both the rate of electrophoresis and the stability of the suspended particle are highly dependent on the magnitude of the Zeta potential. 
The success of an electrokinetic treatment depends largely upon the amount of particles that can remain stable and suspended long enough to penetrate well into the porous network of the HCP [11]. Particles that fall out of suspension or that collapse into a gel prematurely cannot be transported into the pores. The stability of a suspension of particles mainly depends upon the Zeta potential, which in turn is dependent upon the fluid $\mathrm{pH}$, and the presence of stabilizing ions [14] [15] [16]. Particle characteristics that are also important in stabilizing the suspension include the particle size and concentration, and its affinity for ionic stabilizing agents that can be used to influence the Zeta potential [17] [18] [19]. The suspension temperature also influences stability [20].

Suspended particle instability can manifest as coagulation [14] [21]. This term applies to situations in which particles collide and stick to each other, forming flocs [21]. Coagulated particle flocs can fall to the bottom of the fluid chamber, as a sediment. In other cases, the coagulation is rapid and pervasive enough to cause the fluid suspension to suddenly transform into a semi-solid gel. When coagulation is caused by electrochemically manipulating the Zeta potential, it can be considered electro-coagulation. In the water and wastewater treatment industry, electro-coagulation is practiced for control of suspended solid. This method involves electrolytic addition of coagulating contaminants. These electro-coagulation processes have also been generated by activation of sacrificial anodes. Ions generated from these anodes are used to promote coagulating chemical interactions allow efficient pollutant removal by sedimentation or filtration [22] [23]. In some cases, electro-coagulation can be achieved by electrophoretically driving particles toward a mesh filter that collects them for removal. The efficiency of these other electro-coagulation processes can be enhanced by $\mathrm{pH}$-induced reductions of the magnitude of the Zeta potential.

As mentioned earlier, $\mathrm{pH}$ plays a significant role in particle stability. During a given treatment, $\mathrm{pH}$ changes can be induced by the treatment electrodes. At the anode, the oxidation of water as follows:

$$
2 \mathrm{H}_{2} \mathrm{O} \rightarrow \mathrm{O}_{2}+4 \mathrm{H}^{+}+4 \mathrm{e}^{-}
$$

This reaction will produce $\mathrm{H}+$ (hydrogen ions) at the anode. $\mathrm{H}+$ ions located adjacent to the treatment anode will tend to reduce the $\mathrm{pH}$ value in this vicinity [24].

At the cathode, the following reaction will produce $\mathrm{H}_{2}$ (hydrogen gas) and $\mathrm{OH}^{-}$(hydroxide ions) [24].

$$
2 \mathrm{H}_{2} \mathrm{O}+2 \mathrm{e}^{-} \rightarrow \mathrm{H}_{2}+2 \mathrm{OH}^{-}
$$

Another influence on the $\mathrm{pH}$ in the treatment fluid is the absorption of atmospheric carbon dioxide. When carbon dioxide dissolves into the suspension fluid, it will react as follows:

$$
\mathrm{CO}_{2}+\mathrm{H}_{2} \mathrm{O} \rightarrow \mathrm{H}^{+}+\mathrm{HCO}_{3}^{-} \rightarrow 2 \mathrm{H}^{+}+\mathrm{CO}_{3}^{2-}
$$

where $\mathrm{CO}_{2}$ is the carbon dioxide, $\mathrm{HCO}_{3}^{-}$is bicarbonate ion, $\mathrm{H}_{2} \mathrm{CO}_{3}$ is carbonic acid, and $\mathrm{CO}_{3}^{2-}$ is the carbonate ion [25]. With the absorption of carbon dio- 
xide, the $\mathrm{pH}$ value in the fluid drops.

Although not widely reported in the literature, coagulation can commonly be observed during successful electrokinetic particle treatments. In considering the several factors that can influence electro-coagulation, the strength of the applied E-field may play a significant role. It is conceivable that a sufficiently high electric field can cause suspended particles to crowd together just prior to entering a given pore and thus suffer an increased risk of collision and coagulation. This hypothesis is illustrated in Figure 1. When no E-field is applied, particles tend to wander about randomly via Brownian motion. They remain separated from a stable suspension due to mutual electrostatic repulsion. When a low E-field is applied, it can drive these particles to penetrate the pores in sequence, thus permitting them to reduce the porosity of the cement. When the applied E-field is high, it can force the particles into relatively close proximity where a higher collision risk can cause them to coagulate.

These electro-coagulated particles can tend to collect adjacent to the cement surfaces and block some of the pore openings. The main emphasis of this study was to examine the role of E-field strength and $\mathrm{pH}$ changes that can influence particle stability and thus treatment efficiency. This work involved an effort to identify a threshold value of the E-field that causes particle instability.

\section{Research Significance}

Electrokinetic nanoparticle treatments have been shown to exhibit remarkable benefits for mitigating reinforcement corrosion, increasing concrete strength, and reversing the effects of sulfate attack. In these studies, and in pilot field applications particle stability had become a significant barrier for achieving efficient and reproducible outcomes [8] [12]. Unstable particles tend to fall out of suspension. This halts particle transport. In the current study key stability parameters were defined. Vital threshold values were identified that protect particle stability while ensuring effective transport. This achievement is expected to greatly expand the range of treatment applications that this technology will enable.
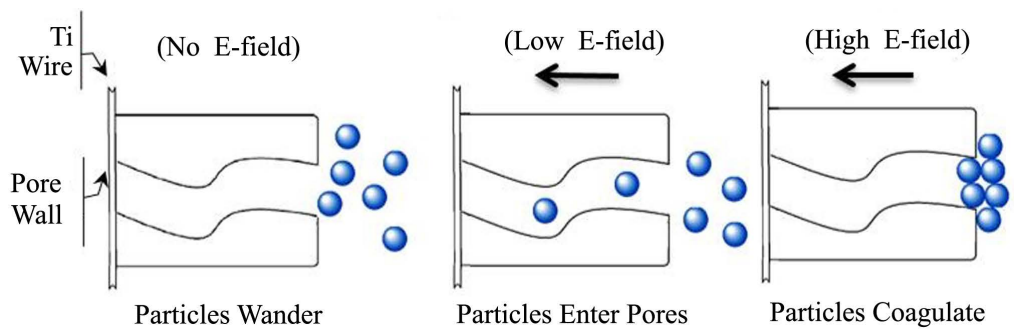

Figure 1. Particles in a stable suspension normally repel each other. They wander about randomly due to Brownian motion. Under an applied electric field, the particles are forced toward the cement pores. Under a low E-field, the particles are able to remain separated and penetrate the pores in sequence. Under a high E-field, particles are forced into close proximity where they are at higher of risk collision and may possibly coagulate at the pore openings. 


\section{Experiential Procedure}

Figure 2 illustrates the dimensions of the cylindrical hardened cement paste specimens used in this study. Care was taken to ensure that the bottom end of the embedded titanium wire was equidistant from the bottom and the side surfaces of each specimen. This was done to facilitate a reasonably uniform electric field throughout each cement specimen during electrokinetic treatment.

The specimens consisted of ordinary Type I Portland cement (Ash Grove Cement Company, Little Rock, AR) with a 0.48 water-cement ratio. Table 1 lists the chemical composition. The mixer (KitchenAid, 6-liter, Classic Model, Whirlpool Corporation, Greenville, Ohio) used to blend each batch was operated at low speed. Batching procedures followed the ASTM C 305 standard. 24 hours after laboratory batching, the specimens were demolded and lime water cured for two weeks.

In order to determine the threshold E-field value that could cause electro-coagulation of particles as they approached the cement pores, a series of test treatments were conducted on individual specimens as shown in Figure 3. Observations of coagulation were recorded for cases involving both negatively and positively charged silica nanoparticles (commercially available products and obtained from NALCO Water Company, Naperville, IL). The negatively charged

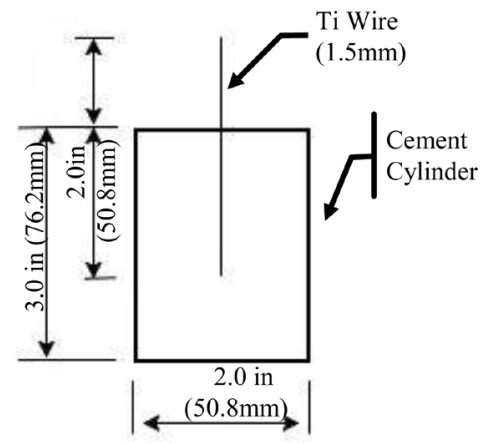

Figure 2. Dimensions of hardened cement paste specimens. 1 inch equals to $25.4 \mathrm{~mm}$.

Table 1. Mill test result of type I (Low Alkali) cement powder used in this study.

\begin{tabular}{cc}
\hline Component & Amount (mass \%) \\
\hline $\mathrm{CaCO}_{3}$ & 2.41 \\
$\mathrm{SiO}_{2}$ & 20.15 \\
$\mathrm{Al}_{2} \mathrm{O}_{3}$ & 4.62 \\
$\mathrm{Fe}_{2} \mathrm{O}_{3}$ & 4.03 \\
$\mathrm{CaO}$ & 63.61 \\
$\mathrm{SO}_{3}$ & 3.20 \\
$\mathrm{Na}_{2} \mathrm{O}$ & 0.16 \\
$\mathrm{~K}_{2} \mathrm{O}$ & 0.57 \\
$\mathrm{CO}_{2}$ & 1.08
\end{tabular}




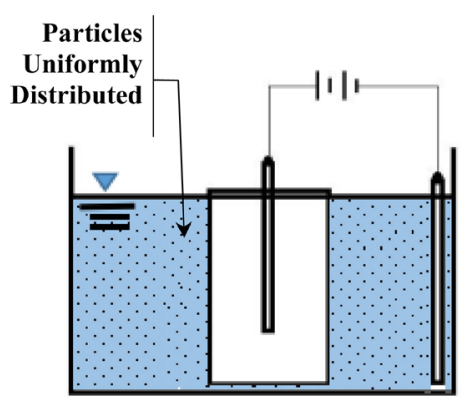

Figure 3. Individual specimen within an electrokinetic nanoparticle treatment setup.

particle suspension was NalCo 1050 Silica sol, with a 20-nm particle. The positively charged particle suspension was NalCo 1056, alumina-coated, silica sol with a $24-\mathrm{nm}$ particle. Each test was run continuously over a $4-6$ day period with the E-field being adjusted daily. During these treatment periods the particle suspensions were removed and replaced with a fresh batch of particles on a daily basis. The volume dilution ratio for each suspension batch was 4 parts sol to 100 parts of deionized water.

The preliminary test treatments were used to determine the threshold E-field for avoiding electro-coagulation. More extensive treatments were conducted later to study particle stability, the resultant cement porosity, and the strength results due to treatment. Two treatment categories were established for these more extensive treatments based upon their respective parameters; the current control group and the E-field control group. The specimens of the current control group were treated with a current density that was limited to $1.5 \mathrm{~A} / \mathrm{m}^{2}$ (the ACI damage threshold) [26]. For the E-field control group, the treatment was conducted below the electro-coagulation threshold E-field value that was identified during the preliminary phase of the study. The treatment circuit and setup for both of these treatment groups is shown in Figure 4.

The positive pole of the power supply was connected to the titanium mesh electrode that was immersed in the suspension fluid surrounding the cement specimens. This polarity drove the positively charged sol particles toward the titanium wire that were embedded within each cement specimen. The negative pole of the power supply was connected to the titanium wires that were centered within the cement specimens. During these treatments, the initial volume dilution ratio was the same as had been used for the preliminary tests (4 parts soil to 100 parts of deionized water). One difference in these cases was that the full particle dosage was provided incrementally on a daily basis without removing any portion of the treatment fluid from the prior day. The overall dosage was calculated based upon the available capillary volume porosity that could receive particles. In these treatments, based on a $20 \%$ volume porosity assumption, a $30-\mathrm{cm}^{3}$ volume of pores was estimated for each specimen. This pore volume was targeted to receive $30 \mathrm{~cm}^{3}$ of particles. Compressive strength tests were conducted in accordance with ASTM C873 directly after treatment. Prior to capping the cement cylinders, the exposed portions of the embedded titanium electrodes 


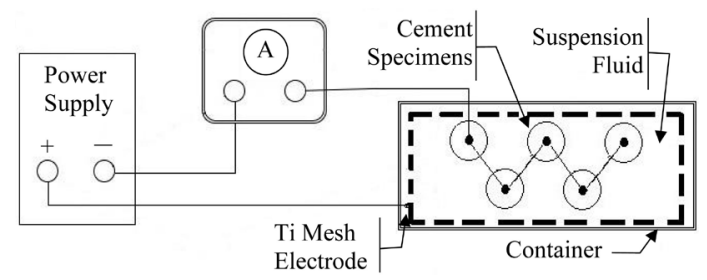

Figure 4. Top view of treatment circuit setup for electrokinetic nanoparticle treatment of hardened cement paste cylinders.

were trimmed flush with the top surfaces of the specimens. After compressive strength testing, specimen fragments were subjected to evaporate porosity measurement utilizing $105^{\circ} \mathrm{C}$ to drive out resident moisture.

$\mathrm{pH}$ monitoring of the particle suspension fluid was conducted throughout the treatment period to assess the potential impact on particle suspension instability. Measurements were conducted at selected locations (as identified in the results section) to examine $\mathrm{pH}$ gradients that could destabilize the suspension during a given treatment.

\section{Results and Discussions}

In order to determine the threshold electric field value that could cause electro-coagulation of particles as they approach the cement pores a series of tests was conducted on individual specimens as shown in Figure 3. Table 2 shows the observations of coagulation recorded for a negatively charged $20 \mathrm{~nm}$ silica particle. Each test was run continuously over a 6-day period with the electric field being adjusted daily. The particle concentration of the surrounding fluid was reset to the same starting value on a daily basis. For one specimen the electric field was started at a high value of $25.2 \mathrm{~V} / \mathrm{cm}$ and gradually decreased to a low value of $1.77 \mathrm{~V} / \mathrm{cm}$. The large starting E-field value correlated to approximately twice the electric field that could be permitted in accordance with ICRI code [27]. A companion specimen was started at a low value of $0.787 \mathrm{~V} / \mathrm{cm}$ and run to a high value of $3.14 \mathrm{~V} / \mathrm{cm}$.

For the specimen starting at the high value of $25.2 \mathrm{~V} / \mathrm{cm}$, coagulation was observed for every specific potential that was applied throughout the test period. The observation of a "Strong Gel" case of coagulation was noted when the removal of the resulting gel required scrapping of the specimen. An image of a "Strong Gel" gelled specimen is shown in Figure 5. The values of electric field that did not exhibit coagulation were $0.787 \mathrm{~V} / \mathrm{cm}$ and $1.57 \mathrm{~V} / \mathrm{cm}$. All other levels of the applied electric field exhibited significant coagulation on the surfaces of the specimens. As noted in the background section, there are a number of possible causes for particle coagulation. The current study encountered two competing causes, high E-field strength and shifting $\mathrm{pH}$ levels within the treatment fluids. When coagulation appeared, it was found on each specimen surface as well as on the bottom of the treatment chamber (directly adjacent to each specimen).

In Table 2 the specimen subjected to a decreasing electric field exhibited serious coagulation at every electric field level in the range of 25.2 to $2.76 \mathrm{~V} / \mathrm{m}$. 


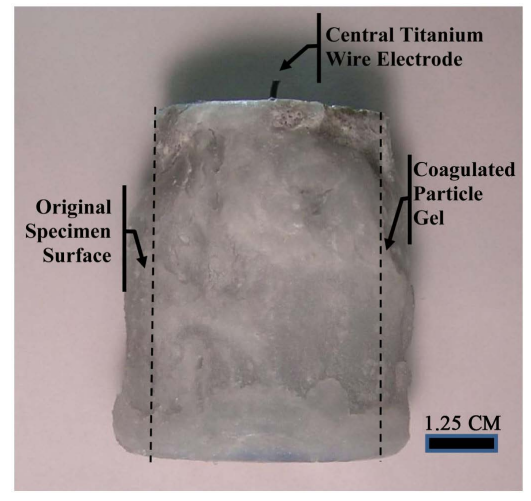

Figure 5. Day 1. Specimen subjected to a decreasing electric field. 20-nm (negatively charged) Silica sol treatment, was applied with a starting electric field of $25 \mathrm{~V} / \mathrm{cm}$. See Table 2. Submerged portions of the specimen developed a gel covering of electro-coagulated particle.

Table 2. Electro-coagulation observation of $20-\mathrm{nm}$ silica sol with negative charge.

\begin{tabular}{cccccc}
\hline \multirow{2}{*}{ Trial } & \multirow{2}{*}{ Day } & \multicolumn{2}{c}{ Decreasing Electric Field } & \multicolumn{2}{c}{ Increasing Electric Field } \\
\cline { 3 - 6 } & & $\mathrm{E}(\mathrm{V} / \mathrm{cm})$ & Coagulation Observed & $\mathrm{E}(\mathrm{V} / \mathrm{cm})$ & Coagulation Observed \\
\hline 1 & 1 & 25.2 & Strong Gel & 0.787 & None \\
2 & 2 & 12.6 & Strong Gel & 1.57 & None \\
3 & 3 & 6.3 & Strong Gel & 3.14 & Strong Gel \\
4 & 4 & 2.76 & Strong Gel & - & - \\
5 & 5 & 2.24 & Weak Gel & - & - \\
6 & 6 & 1.77 & Weak Gel & - & - \\
\hline
\end{tabular}

a. Removal of coagulation gel required scrapping. b. Removal of coagulation gel only required rinsing.

Coagulation was also appearing at electric fields as low as 2.24 and $1.77 \mathrm{~V} / \mathrm{m}$. During the course of this test series it was likely that coagulating particles may have provided significant blockage of pore openings that would not have been reopened simply due to mechanical scraping. While this would have limited the number of pores available to receive particles, this did not mean that the lower concentration of pore openings would necessarily produce additional coagulation or significantly influence the threshold electric field value. This is because a sufficiently low electric field would not have produced electro-coagulation regardless of the concentration of particles or the number of available pore openings. Instead of coagulating, it was most likely that these particles would form a traffic jam. They would probably have simply held their positions in the fluid suspension (as controlled by the mutual electrostatic repulsion). As a result, it would have been expected that a field value below the threshold would have allowed the particles to crowd together without colliding. Continuously reducing the number of available pore openings would not have caused the crowd of particles to start colliding, unless the electric field was being magnified in the vicinity of these pores openings. Such magnification was unlikely since the availability of ions (forming via hydrolysis) was relatively steady. In light of these cir- 
cumstances, observing a threshold electric field value would not appear to be significantly dependent upon whether the field was increasing or decreasing during the evaluation process. Table 2 shows that the threshold value for the electric field was in the range of $1.77-1.57 \mathrm{~V} / \mathrm{m}$. Based on these observations, it appears that the same threshold value for electro-coagulation was achieved regardless of whether the applied electric field was increasing or decreasing as this threshold value was being approached.

Similar to Table 2, Table 3 shows the electro-coagulation test observations recorded for a positively charged, 24-nm, alumina-coated, silica particle. These results were used to determine the threshold electric field value for avoiding electro-coagulation at the surface of cement specimens. Over a 4-day test period, two treatment cases were applied. In the first case, the specimen was subjected to a broadly ranging electric field $(25.2$ to $1.77 \mathrm{~V} / \mathrm{cm})$. In the other case, the specimen was subjected to a relatively low ranging electric field (0.787 to 0.400 $\mathrm{V} / \mathrm{cm}$ ). This electric field was adjusted daily. The treatment suspension was also renewed daily to re-establish the original starting concentration. For each treatment case, the starting electric field value was at the high end of the test range. In most cases the observed coagulation was found to be either just "Weak Gel" or "Strong Gel". When the electric field value was reduced to $0.4 \mathrm{~V} / \mathrm{cm}$ no coagulation was observed. The specimen surface was free of coagulated particles as shown in Figure 6.

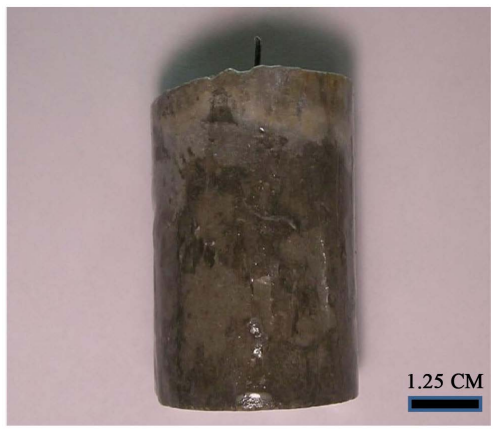

Figure 6. Day-4. Specimen subjected to a low ranging electric field. 24-nm, positively charged, alumina-coated, silica sol treatment, was applied with a starting electric field of $0.4 \mathrm{~V} / \mathrm{cm}$. See Table 3. Submerged portions of the specimen were not covered with coagulated particles.

Table 3. Electro-coagulation observations of $24 \mathrm{~nm}$ alumina-coated silica sol with positive charge.

\begin{tabular}{cccccc}
\hline \multirow{2}{*}{ Trial } & \multirow{2}{*}{ Day } & \multicolumn{2}{c}{ Broad Ranging Electric Field } & \multicolumn{2}{c}{ Low Ranging Electric Field } \\
\cline { 3 - 6 } & & $\mathrm{E}(\mathrm{V} / \mathrm{cm})$ & Coagulation Observed & $\mathrm{E}(\mathrm{V} / \mathrm{cm})$ & Coagulation Observed \\
\hline 1 & 1 & 25.2 & Strong Gel $^{\mathrm{a}}$ & 0.787 & Strong Gel \\
2 & 2 & 1.18 & Strong Gel & 0.787 & Strong Gel \\
3 & 3 & 0.59 & Weak Gel & 0.40 & None \\
4 & 4 & 0.59 & Weak Gel & 0.40 & None \\
\hline
\end{tabular}

a. Removal of coagulation gel required scrapping. b. Removal of coagulation gel only required rinsing. 
In Table 3, coagulation was observed on both specimens at every applied electric field in the range of $25.2 \mathrm{~V} / \mathrm{cm}$ to $0.59 \mathrm{~V} / \mathrm{cm}$. For the low ranging electric field experiment, "Strong Gel" electro-coagulation was observed after applying an electric field value of $0.787 \mathrm{~V} / \mathrm{cm}$ on Day 1 . In order to examine this behavior further, this electric field value was applied a second time on Day 2. "Strong Gel" gelling was again observed following Day 2. Similarly, the $0.4 \mathrm{~V} / \mathrm{cm}$ electric field was also repeated. In this case, both trials exhibited no electro-coagulation. Comparing these threshold values for the 24-nm alumina-coated silica particles and the $20-\mathrm{nm}$ silica particles, it appears that the threshold value for the positively charged 24-nm alumina-coated silica particles (which was $+0.4 \mathrm{~V} / \mathrm{cm}$ ) was significantly smaller than that of the negatively charged $20-\mathrm{nm}$ silica particles (which was $-1.57 \mathrm{~V} / \mathrm{cm}$ ).

As noted earlier, it was conceivable that the particles applied on the Day-1 trial could have caused some amount of pore blocking, so as to influence the threshold E-field findings of later trials. This is unlikely since coagulation can occur when the particle concentration is high enough to cause a backlog of particles waiting to enter a pore. A sufficiently high E-field is expected to cause these particles to collide and coagulated as they wait their turn to enter the pore. A limited or even diminishing number of pore openings would not be expected to change this situation significantly. It is anticipated that the reduction in available pore openings caused by prior coagulation events would not be expected to alter the observed threshold E-field significantly. The findings of Table 3 indicate a threshold E-field of approximately $0.4 \mathrm{~V} / \mathrm{cm}$ regardless of the amount of coagulation that was exhibited in the early trials. Based on these observations, it appears that the particles would tend to avoid coagulation regardless of how limited the remaining number of pore openings may become.

Figure 7 and Figure 8 contain results for treatments conducted above and below the electric field (E-field) threshold value of $0.4 \mathrm{~V} / \mathrm{cm}$, that was found to cause electro-coagulation of the 24-nm alumina-coated silica particle. One group of specimens were treated above the $0.4 \mathrm{~V} / \mathrm{cm}$ threshold, but were limited to a maximum current density of $1.5 \mathrm{~A} / \mathrm{m}^{2}$ (as per International Concrete Repair Institute code), to avoid strength loss in the cement [26]. This limiting current density was maintained with an electric field that ranged from 0.9 to $0.75 \mathrm{~V} / \mathrm{cm}$. This treatment category is referred to as the current control group. These specimens were treated for two weeks. The full particle dosage was provided incrementally on a daily basis. This dosage was calculated based on the estimated available capillary volume porosity that could receive particles. In this case, 150 $\mathrm{cm} 3$ of particles was provided for an estimated $150 \mathrm{~cm}^{3}$ volume of pores. The actual pore volume available for treatment may vary due to the degree of pore continuity as influence by weathering factors. Since the particle packing efficiency typically averages in the vicinity of $50 \%$, the dosage provided was significantly in excess of the available pore volume [28]. Electro-coagulation was observed on each day of the treatment period for the current control group. 


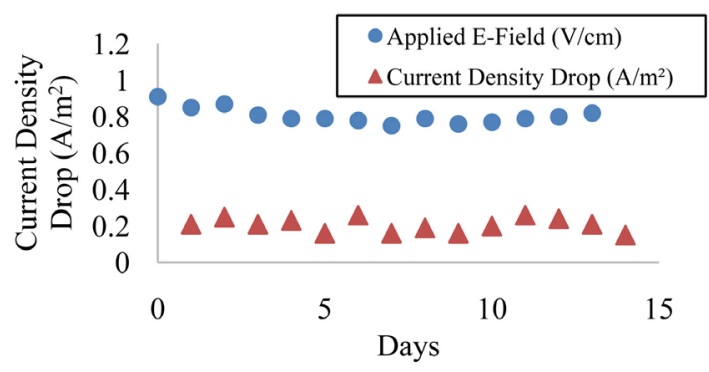

Figure 7. Current density drop plot for $24 \mathrm{~nm}$ Alumina-Coated Silica Sol treatment under current control. The treatment under current control resulted in significant electro-coagulation. The applied voltage for this treatment adjusted daily to limit current to $1.5 \mathrm{~A} / \mathrm{m}^{2}$ to avoid strength loss, and the total sum of daily current drops was $2.9 \mathrm{~A} / \mathrm{m}^{2}$.

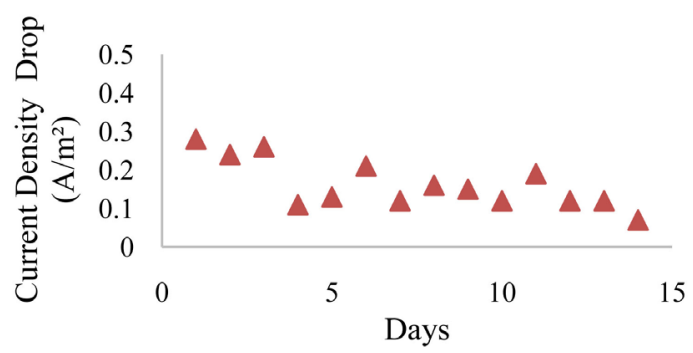

Figure 8. Current density drop plot for $24 \mathrm{~nm}$ Alumina-Coated Silica Sol treatment under E-field control. The treatment under E-field control resulted in no significant electro-coagulation. The applied voltage limited to $0.4 \mathrm{~V} / \mathrm{cm}$ to avoid suspension instability, and the total sum of daily current drops was $2.3 \mathrm{~A} / \mathrm{m}^{2}$.

Figure 5 contains an example of a specimen exhibiting a gel coating of the coagulated particles. This coagulated particle gel was scrapped from each specimen surface following each day of treatment. Gel scraping may only be possible for localized field applications such as the treating of cracks. It was done here in an attempt to sustain the availability of open pores that could allow particles to continue entering the cement as the treatment continued. A drop in current density was observed during each day of the treatment period. The magnitude of this current density drop is plotted in Figure 7. This drop was recorded prior to the daily scrapping of coagulated particles and the dosing of additional particles. The total accumulated current drop came to $2.9 \mathrm{~A} / \mathrm{m}^{2}$.

The current density control group (Figure 7) was subjected to an electric field value that ranged from $0.82 \mathrm{~V} / \mathrm{cm}$ to $0.9 \mathrm{~V} / \mathrm{cm}$, which was twice the threshold value $(0.4 \mathrm{~V} / \mathrm{cm}$ observed from Table 3$)$. For this reason, it was not surprising that coagulation was observed. This high E-field appeared to provide a relatively excessive electrostatic force for driving the particles. The relatively high concentration of particles adjacent to each pore opening would have induced electrostatic interference with each other's' progress, as they were entering a given pore. Due to the high driving force of the E-field, this electrostatic repulsion would not have been sufficient to keep them from being driven close enough to collide and coagulate (this is illustrated in Figure 1). Due to this instability, relatively 
few particles tended to enter the pores prior to coagulating and falling out of suspension. Because of this high collision frequency within the cloud of particles adjacent to each pore opening, many particles would have been unable to remain suspended. This region of high particle collision frequency tended to produce a coagulated gel on the specimen surfaces just outside of the pore openings (see Figure 1). The benefit of a given treatment depended upon the number of particles that could penetrate the pores. It appeared that relying solely on the maximum current density value to control a treatment was inappropriate since it did not protect against electro-coagulation losses.

In contrast to the current density control group, another 24-nm alumina-coated silica treatment was conducted for the E-field control group. These results are shown in Figure 8. As noted earlier, the E-field threshold value for this treatment $(0.4 \mathrm{~V} / \mathrm{cm})$ was determined from the preliminary testing that is summarized earlier in Table 3. Using this threshold value as the applied electric field was done to avoid electro-coagulation and the associated loss of particles. The treatment period and overall dosages were held to the same values as were used earlier for the current control group (Figure 7). The current density in this treatment period was governed by the E-field that was being applied. In this case the current density ranged from $0.91 \mathrm{~A} / \mathrm{m}^{2}$ to $0.59 \mathrm{~A} / \mathrm{m}^{2}$.

Since the threshold E-field value was determined from the preliminary testing reported in Table $3(0.4 \mathrm{~V} / \mathrm{cm})$, an E-field controlled treatment would be expected to provide a functional treatment option. Using a threshold E-field to control treatment, the applied E-field would be expected to hold particles stable in the suspension, awaiting their opportunities to enter into available pores, sequentially. This became evident when a concentrated particle cloud formed during treatment as illustrated on the right side of Figure 9. A relatively few of the particles suffered coagulation as reported in Table 3 (at $0.4 \mathrm{~V} / \mathrm{cm}$ ). These particles in the suspension cloud were relatively stable. As a result, they were not tending to form coagulated blockages of the pore openings on the cement surfaces. The penetration of particles into the pores would have proceeded relatively unhindered. Based on these observations, a maximum E-field threshold value utilized in a given treatment may help minimize coagulated particle losses.

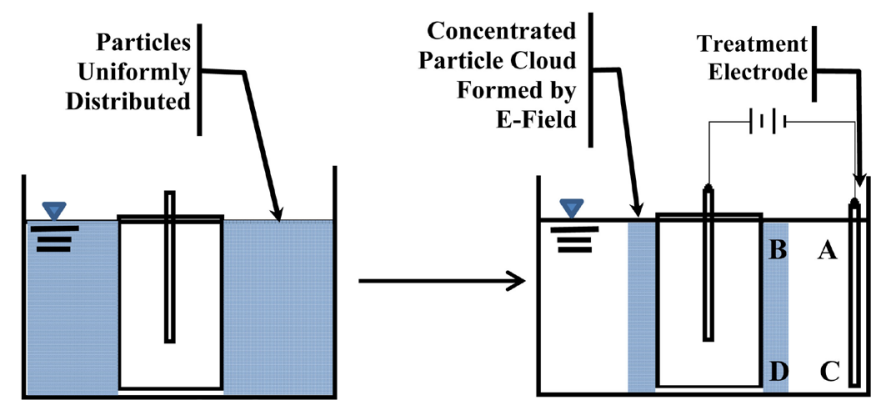

Figure 9. After applied the appropriate electric field, the distribution of particles changed from uniformly to concentrated cloud. Concentrated cloud of particles is waiting for the turn to enter specimen surface pores. Location A-D were the $\mathrm{pH}$ monitoring sites. 
Specimens treated with 24-nm alumina coated silica were subjected to strength and porosity testing. These results are presented in Figure 10. The results were obtained for 3 groups based on their treatment parameters; the E-field control group, the current control group, and the untreated control group. For the current control group, the applied E-field value ranged from 0.7 to $0.9 \mathrm{~V} / \mathrm{cm}$. An average E-field value of $0.8 \mathrm{~V} / \mathrm{cm}$ was reported for this group in Figure 10. For the E-field control group, the highest strength value observed was $5 \mathrm{ksi}$. The porosity results for this group averaged $21 \%$. For the current control group, the strength test results ranged as high as $4 \mathrm{ksi}$. The porosity values for this group averaged $23 \%$. This group exhibited electro-coagulation. For the untreated control group, the porosity averaged $26 \%$. The peak strength test result for this group was $3 \mathrm{ksi}$.

Student $\mathrm{F}$ and $\mathrm{T}$ test were selected to prove that all porosity test were provided to statistically demonstrate that these porosity results of Figure 10 exhibited significant differences when comparing one case to another. These calculations were conducted in accordance with "Exploring Chemical Analysis" [29]. After comparing the results for the E-field control group, the current control group and the untreated group, each of these test cases was found to exhibit statistically significant differences.

From Figure 10, the average compression strength results were $2.2 \mathrm{ksi}$ for the untreated group, $2.8 \mathrm{ksi}$ for the current density control group and $3.2 \mathrm{ksi}$ for the E-field control group. The E-field control group exhibited a nearly $50 \%$ strength increase as compared to the untreated group. For the current density control group, the strength increased by $25 \%$, despite a significant amount of gel formation that inhibited particle delivery. The current density control group and the E-field control group exhibited $23 \%$ and $21 \%$ average porosities (respectively) while the untreated group exhibited the porosity of $26 \%$. As expected the porosity decreases observed in this study were consistent with the strength increases observed among these three study groups. Based on these observations, the particle treatments were found to be effective at increasing the compressive strength and decreasing the porosity even when significant particle instability and losses were occurring during the treatment process.

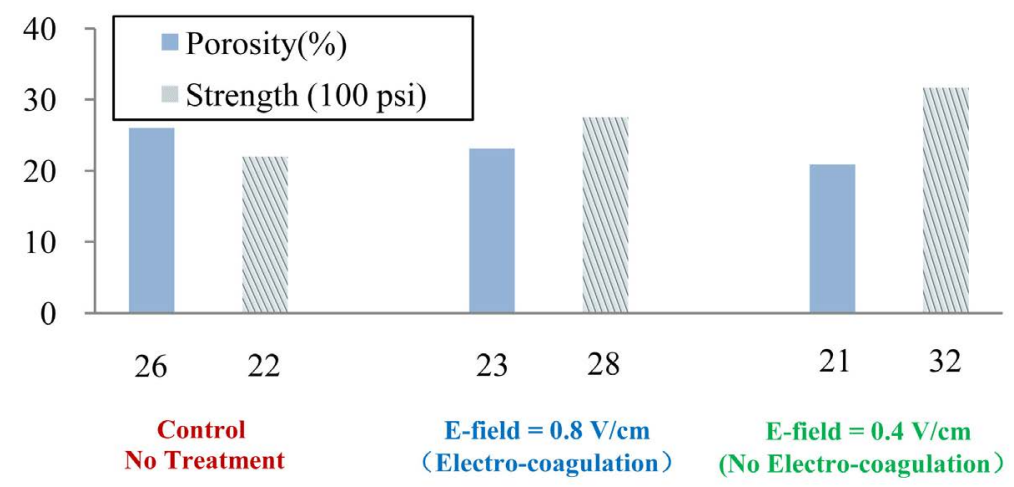

Figure 10. Strength and porosity plot for $24 \mathrm{~nm}$ Alumina-Coated Silica Sol treatment groups and control group. 
In designing the nanoparticle treatment dosage, it was assumed that the entire pore volume of a given specimen was accessible. This assumed continuity of capillary pores throughout the matrix and that the sizes and shapes of each pore were sufficient to permit particle access. In contrast, mature, un-weathered cement paste is expected to exhibit multiple pore sizes, bottle necks, and randomly varying degrees of pore discontinuity. During the treatment period, current drop was observed during each day of treatment. This drop could be attributed to the ion polarization at each electrode, the capture of particles (which are charge carriers) at pore bottle necks, and the increasing circuit resistance as conduction through the pore network was inhibited by trapped particles. During the 2 -week treatment period approximately $60 \%$ of the dosed particles were delivered into the HCP pores. The porosity reductions noted in Figure 10 were observed throughout these specimens, as opposed to being confined to just the surface areas. This suggests that randomly distributed areas of bottle necks and discontinuity were trapping particles at various locations throughout the HCP matrix.

Based on the average strength and the average porosities reported in Figure 10 , the benefits achieved in the E-field control group were almost twice the value of those exhibited by the current density control group. The E-field control group was driven by a relatively low threshold value $(0.4 \mathrm{~V} / \mathrm{cm})$, which was one-half the field strength used for the current control group. For this reason, more particles escaped coagulation and were able to penetrate into the cement pores under E-field control. They were available in relatively large numbers that enabled the observed reductions in porosity and the increased strength. In contrast, the high E-field $(0.8 \mathrm{~V} / \mathrm{cm})$ of the current control group tended to coagulate some of the suspended particles. The coagulated particles were lost and unavailable for entering the pores. This indicated that the treatments may be rendered more effective by maintaining an applied E-field that is below the threshold value $(0.4 \mathrm{~V} / \mathrm{cm})$. Based on these observations it appears that an appropriate electric field value, which does not cause electro-coagulation, would produce greater mechanical benefits for a given particle treatment of ordinary Portland cement.

Daily $\mathrm{pH}$ monitoring was conducted in order to determine if $\mathrm{pH}$ changes observed during treatment were influencing coagulation. Four monitoring points were established to evaluate $\mathrm{pH}$ during treatment (see Figure 9). Figure 11 contains these results which were conducted under E-field control at $0.4 \mathrm{~V} / \mathrm{cm}$. For the first 8 days, a cloud of particles appeared within a centimeter of the specimen surface. This cloud of particles was easily re-dispensable by agitation. During the entire treatment period the $\mathrm{pH}$ increased at each of the monitoring points. It did not reach the $\mathrm{pH}$-induced suspension collapse threshold which occurs at approximately 5 to 5.5 for this sol. After 8 days of treatment, particles started to exhibit some slight gelling. This meant that the particles in the gel could not be redisposed by agitation. During this same period, the $\mathrm{pH}$ values continued to increase. At the fluid surface adjacent to the treatment electrode 


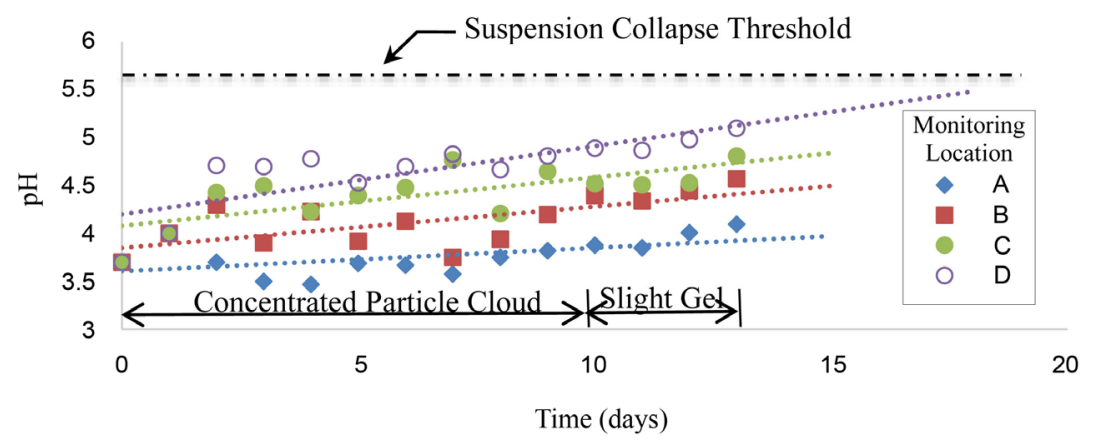

Figure 11. $\mathrm{pH}$ monitoring of $24 \mathrm{~nm}$ Alumina-Coated Silica Sol treatment under E-field Control $(0.4 \mathrm{~V} / \mathrm{cm})$. When extrapolated, suspension collapse (at $\mathrm{pH}=5.5$ ) is predicted; A-Day 77, B-Day 38, C-Day 28, and D-Day 18. Each curve fit exhibited a 99\% confidence. Concentrated particle cloud (Not gelled) was observed adjacent to the cement specimen surface. The rest of the suspension fluid was clear. See illustration in Figure 10.

(Location A), the $\mathrm{pH}$ increased from 3.8 to 4.1 . The $\mathrm{pH}$ increase at the fluid surface adjacent to the specimen (Location B) was from 3.8 to 4.6. At the bottom of the treatment container, the $\mathrm{pH}$ value adjacent to the treatment electrode (Location C) increased from 3.8 to 4.8. Adjacent to the specimen (Location D), this increase went from 3.8 to 5.2. By comparison, the initial $\mathrm{pH}$ within the HCP specimens was probably in the vicinity or 13.6 to 12.4 as influenced by the 2 -week, limewater curing period that concluded just prior to the start of treatment.

As contained in Figure 11, some slight gel formation was appearing after 9 days of the treatment period. This small amount of instability may have been influenced by the amount of suspended particles that had been accumulating as a dense cloud (see Figure 9) during the trial. As the concentration of particles in this cloud kept increasing, the average distance between particles was reducing. This tended to cause the probability of particle collisions to increase. The slight amount of gelling incurred could be associated with the elevated concentration of particles present in this concentrated cloud that formed during treatment. After this slight gel started to appear, the $\mathrm{pH}$ values appeared to follow a higher slope as the system approached the $\mathrm{pH}$-induced suspension collapse threshold value of 5.5. It is conceivable that the true behavior of the particle system may be better modeled with two straight lines. Future work will examine this possibility. At Day 11, the highest $\mathrm{pH}$ value was 4.9 at monitoring location D. This suggests that all observed coagulation was electro-coagulation from Day 9 to Day 11. On Days 12 and 13, the $\mathrm{pH}$ values at location D (5.0 and 5.1) had reached very fairly to the suspension collapse since the threshold value is typically in the range of 5 to 5.5. This suggests the possibility that some amount of $\mathrm{pH}$-induced coagulation might have been occurring at location D. Based on these observations, the $\mathrm{pH}$ values did not appear to significantly affect coagulation during the E-field control treatment.

As shown in Figure 11, $\mathrm{pH}$ values immediately started to differ among each of the monitoring points $(\mathrm{A}-\mathrm{D})$. The $\mathrm{pH}$ values exhibited a gradient in two direc- 
tions. In the horizontal direction (From $\mathrm{B}$ to $\mathrm{A}$ and from $\mathrm{D}$ to $\mathrm{C}$ ), the $\mathrm{pH}$ differed by as much as 0.5 by Day 13. In the vertical direction (From $B$ to $D$ and from $\mathrm{A}$ to $\mathrm{C}$ ), the $\mathrm{pH}$ differed by as much as 1.0. The particles appeared to remain in stable suspension as they approached the cement pore openings, probably due to the mutual electrostatic repulsion and the low E-field. At the cement surfaces, a concentrated particle cloud had formed after Day 1 (see Figure 12). The relatively large amount of suspended particles within this dense cloud area appeared to remain stable. At the same time, the oxidation of water would have been producing $\mathrm{H}^{+}$ions at the external treatment electrode (Anode). It makes sense that $\mathrm{H}^{+}$ions forming adjacent to the treatment electrode would tend to reduce the $\mathrm{pH}$ value in this area (Location $\mathrm{A}$ and $\mathrm{C}$ ) as compared to the Locations $\mathrm{B}$ and $\mathrm{D}$. This difference is noted in Figure 12. For the $\mathrm{pH}$ gradient vertical direction, at the fluid surface, $\mathrm{CO}_{2}$ was available to dissolve into the suspension fluid and react to form $\mathrm{H}_{2} \mathrm{CO}_{3}$. The resulting $\mathrm{HCO}_{3}^{-}$and $\mathrm{CO}_{3}^{2-}$ ions would be accompanied by an increase in $\mathrm{H}^{+}$[25]. This probably explains why the $\mathrm{pH}$ values at the bottom levels (C and D) were generally higher than at the fluid surface (A and $\mathrm{B}$ ). It is conceivable that some agitation could remedy these two $\mathrm{pH}$ gradients and thus delay the $\mathrm{pH}$ rise toward the suspension collapse threshold $(\mathrm{pH}$ $=5$ to 5.5). Similar $\mathrm{pH}$ gradients were observed for the current control treatment cases as summarized in Figure 11.

Figure 12 contains the $\mathrm{pH}$ monitoring results for the current control treatment. The same monitoring points were used in this case as before. During the first 6 days, particles were forming a "weak gel" on the specimen surfaces. At the same time, the surrounding suspension fluid was found to be clear after each treatment day. After the first 6 days of treatment, a strong gel of coagulated particles started to appear daily. This observation was again accompanied by a clear suspension fluid. During the same period, the $\mathrm{pH}$ values at Location D shifted from 4.5 to 5.2, which was approaching the suspension collapse threshold value (5.5). Over the entire (13-Day) treatment period, the $\mathrm{pH}$ values at the fluid

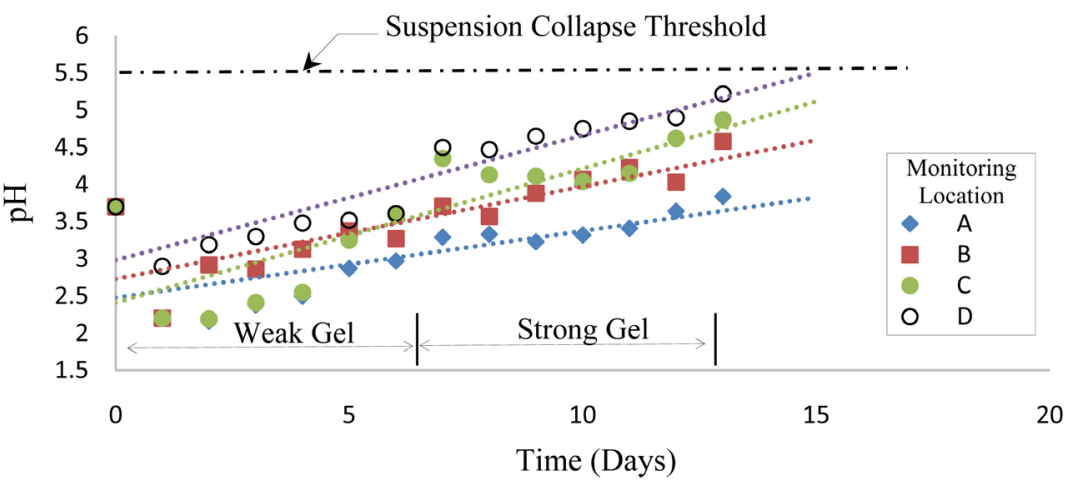

Figure 12. $\mathrm{pH}$ monitoring for $24 \mathrm{~nm}$ Alumina-Coated Silica Sol Treatment under Current Control $(0.8 \mathrm{~V} / \mathrm{cm})$. When extrapolated, suspension collapse (at $\mathrm{pH}=5.5$ ) is predicted; A-Day 34, B-Day 22, C-Day 17, and D-Day 15. Each curve fit exhibited a $99 \%$ confidence. Removal of the weak gel only required rinsing with water. Removal of the strong gel required mechanical scrapping. 
surface (adjacent to the treatment electrode, Location A) increased from 2.2 to 3.7. At the fluid surface (adjacent to the specimen, Location $\mathrm{B}$ ) the $\mathrm{pH}$ increased from 2.2 to 4.4 . Along the bottom of the treatment chamber (nearly $8 \mathrm{~cm}$ from the surface), the $\mathrm{pH}$ values adjacent to the treatment electrode (Location $\mathrm{C}$ ) went from 2.2 to 4.5 . The $\mathrm{pH}$ values adjacent to the specimen (Location $\mathrm{D}$ ) increased from 2.9 to 5.2 .

In general, $\mathrm{pH}$ values observed during the first 6 days in the current control treatment (Figure 12) were lower than the E-field control treatment (Figure 11). The reason for this may be related to the weak gel that was forming at the specimen surface after each day (caused by the high E-field, $0.8 \mathrm{~V} / \mathrm{cm}$ ). The $\mathrm{H}+$ ions (produced by the oxidation of water) were probably being inhibited from entering the cement pores by the "weak gel" at the cement surfaces. This suggests that the high E-field was causing the gelling as opposed to the rise in $\mathrm{pH}$, since these values were in the vicinity of 2.5 to 3.5 (well below the $\mathrm{pH}$-induced coagulation threshold of 5.5). After each day of treatment, the surrounding fluid was clear and apparently free of particles. After the first 6 days of treatment, coagulation was observed as a "strong gel" and $\mathrm{pH}$ values jumped significantly by as much as $1 \mathrm{pH}$ unit. The mechanism of $\mathrm{pH}$ rise stems from electrode reactions mentioned earlier (See Equation (2)). The reason for this relatively sudden increase was unclear, future work will examine the possibility of various factors. During the entire treatment, since the $\mathrm{pH}$ values remained below the suspension collapse threshold value (5.5) the possibility of $\mathrm{pH}$-induced coagulation was considered unlikely. Based on these observations, it appears that the electro-coagulation can be caused primarily by high E-fields, when the suspension remains below the $\mathrm{pH}$-induced coagulation threshold value.

\section{Conclusions and Recommendations}

This study explored several developments for stable and effective electrokinetic treatment with suspended particles. Treatment efficiencies made possible by this work will enable the development of feasible approaches for reversing sulfate attack, mitigating reinforcement corrosion, and enhancing re-alkalization processes to the point where existing structures may be upgraded with microstructural properties that could conceivably exceed the as-built condition. Based on the observations from this study, these conclusions and recommendations are summarized.

- There appears to be a maximum E-field threshold value that should be avoided in order to minimize electro-coagulation and the resulting loss of particles.

- The same threshold value for electro-coagulation was achieved regardless of whether the applied electric field was increasing or decreasing as this threshold value was being approached.

- Particles tend to avoid coagulation regardless of how limited the remaining number of open pores may become during a given treatment. 
- The benefit of a given treatment depended upon the number of particles that could successfully penetrate the pores. It appeared that relying solely on the maximum current density value to control treatment effectiveness was insufficient since it did not protect against electro-coagulation losses.

- The particle treatments were found to be effective at increasing the compressive strength and decreasing the porosity even when significant particle instability and losses were occurring during the treatment process.

- Electro-coagulation can be caused primarily by high E-fields, when the suspension remains below the $\mathrm{pH}$-induced coagulation threshold value.

- For the 14-day treatments conducted in this study, the upward drift of $\mathrm{pH}$ values did not appear to significantly affect particle suspension stability until the suspension collapse threshold $(\mathrm{pH}=5.5)$ was approached.

- It is conceivable that some agitation of the particle suspension fluid may be a potential remedy that could eliminate the formation of $\mathrm{pH}$ gradients and thus delay the $\mathrm{pH}$ rise at specimen surfaces.

\section{Conflicts of Interest}

The authors declare no conflicts of interest regarding the publication of this paper.

\section{References}

[1] Mindess, S. and Young, J.F. (1996) Concrete. Prentice-Hall, Englewood Cliffs.

[2] Lageman, R. (1993) Electroreclamation. Applications in the Netherlands. Environmental Science \& Technology, 27, 2648-2650. https://doi.org/10.1021/es00049a003

[3] Kim, Y.Y., Lee, K.M., Bang, J.W. and Kwon, S.J. (2014) Effect of W/C Ratio on Durability and Porosity in Cement Mortar with Constant Cement Amount. Advances in Materials Science and Engineering, 2014, Article ID: 273460. https://doi.org/10.1155/2014/273460

[4] Lian, C., Zhuge, Y. and Beecham, S. (2011) The Relationship between Porosity and Strength for Porous Concrete. Construction and Building Materials, 25, 4294-4298. https://doi.org/10.1016/j.conbuildmat.2011.05.005

[5] Song, H.W., Pack, S.W., Nam, S.H., Jang, J.C. and Saraswathy, V. (2010) Estimation of the Permeability of Silica Fume Cement Concrete. Construction and Building Materials, 24, 315-321. https://doi.org/10.1016/j.conbuildmat.2009.08.033

[6] Bahadori, H. and Hosseini, P. (2012) Reduction of Cement Consumption by the Aid of Silica Nano-Particles (Investigation on Concrete Properties). Journal of Civil Engineering and Management, 18, 416-425. https://doi.org/10.3846/13923730.2012.698912

[7] Cardenas, H., Kupwade-Patil, K. and Eklund, S. (2011) Corrosion Mitigation in Mature Reinforced Concrete Using Nanoscale Pozzolan Deposition. Journal of Materials in Civil Engineering, 23, 752-760. https://doi.org/10.1061/(ASCE)MT.1943-5533.0000194

[8] Cardenas, H., Kupwade-Patil, K. and Eklund, S. (2011) Recovery from Sulfate Attack in Concrete via Electrokinetic Nanoparticle Treatment. Journal of Materials in Civil Engineering, 23, 1103-1112.

https://doi.org/10.1061/(ASCE)MT.1943-5533.0000255 
[9] Cardenas, H. E. and Struble, L. J. (2006) Electrokinetic Nanoparticle Treatment of Hardened Cement Paste for Reduction of Permeability. Journal of Materials in Civil Engineering, 18, 554-560. https://doi.org/10.1061/(ASCE)0899-1561(2006)18:4(554)

[10] Cardenas, H., Alexander, J. and Kupwade-Patil, K. (2010) Field Testing of High Current Electrokinetic Nanoparticle Treatment for Corrosion Mitigation in Reinforced Concrete. Second International Conference on Sustainable Construction Materials and Technologies, Ancona, 28-30 June 2010.

[11] Kanno, J., Richardson, N., Phillips, J., Kupwade-Patil, K., Mainardi, D.S. and Cardenas, H.E. (2009) Modeling and Simulation of Electromutagenic Processes for Multiscale Modification of Concrete. Journal of Systemics, Cybernetics and Informatics, 7, 69-74.

[12] Kupwade-Patil, K., Cardenas, H.E., Gordon, K. and Lee, L.S. (2012) Corrosion Mitigation in Reinforced Concrete Beams via Nanoparticle Treatment. ACI Materials Journal, 109, 617-626. https://doi.org/10.14359/51684159

[13] Cardenas, H.E. (2002) Investigation of Reactive Electrokinetic Processes for Permeability Reduction in Hardened Cement Paste. University of Illinois at Urbana-Champaign, Urbana and Champaign.

[14] Hamley, I.W. (2013) Introduction to Soft Matter: Synthetic and Biological Self-Assembling Materials. John Wiley \& Sons, New Jersey.

[15] Heard, D.H. and Seaman, G.V.F. (1960) The Influence of pH and Ionic Strength on the Electrokinetic Stability of the Human Erythrocyte Membrane. The Journal of General Physiology, 43, 635-654. https://doi.org/10.1085/jgp.43.3.635

[16] Berg, J.M., Romoser, A., Banerjee, N., Zebda, R. and Sayes, C.M. (2009) The Relationship between $\mathrm{pH}$ and Zeta Potential of $\sim 30 \mathrm{~nm}$ Metal Oxide Nanoparticle Suspensions Relevant to In Vitro Toxicological Evaluations. Nanotoxicology, 3, 276-283. https://doi.org/10.3109/17435390903276941

[17] Wiese, G.R. and Healy, T.W. (1970) Effect of Particle Size on Colloid Stability. Transactions of the Faraday Society, 66, 490-499. https://doi.org/10.1039/tf9706600490

[18] Heris, S.Z., Etemad, S.G. and Esfahany, M.N. (2006) Experimental Investigation of Oxide Nanofluids Laminar Flow Convective Heat Transfer. International Communications in Heat and Mass Transfer, 33, 529-535. https://doi.org/10.1016/j.icheatmasstransfer.2006.01.005

[19] Jiang, J.K., Oberdörster, G. and Biswas, P. (2009) Characterization of Size, Surface Charge, and Agglomeration State of Nanoparticle Dispersions for Toxicological Studies. Journal of Nanoparticle Research, 11, 77-89. https://doi.org/10.1007/s11051-008-9446-4

[20] Wiśniewska, M. (2010) Influences of Polyacrylic Acid Adsorption and Temperature on the Alumina Suspension Stability. Powder Technology, 198, 258-266. https://doi.org/10.1016/j.powtec.2009.11.016

[21] Li, T., Zhu, Z., Wang, D., Yao, C. and Tang, H. (2006) Characterization of Floc Size, Strength and Structure under Various Coagulation Mechanisms. Powder Technology, 168, 104-110. https://doi.org/10.1016/j.powtec.2006.07.003

[22] Rahmani, A.R. (2008) Removal of Water Turbidity by the Electrocoagulation Method. Journal of Research in Health Sciences, 8, 18-24.

[23] Khaled, B., Wided, B., Béchir, H., Elimame, E., Mouna, L. and Zied, T. (2019) Investigation of Electrocoagulation Reactor Design Parameters Effect on the Removal of Cadmium from Synthetic and Phosphate Industrial Wastewater. Arabian Journal 
of Chemistry, 12, 1848-1859. https://doi.org/10.1016/j.arabjc.2014.12.012

[24] Ahmad, Z. (2006) Chapter 3-Corrosion Kinetics. In: Ahmad, Z., Ed., Principles of Corrosion Engineering and Corrosion Control, Butterworth-Heinemann, Oxford, 57-119.https://doi.org/10.1016/B978-075065924-6/50004-0

[25] Jones, D.A. (1992) Principles and Prevention of Corrosion. Macmillan, New York City.

[26] Drewett, J. and Broomfield, J. (2011) An Introduction to Electrochemical Rehabilitation Techniques. Technical Notes No. 2, Corrosion Prevention Association, Hampshire, 8.

[27] Schmick, B.L. (1997) Concrete Repair Manual. 2nd Edition, Vol. 2, The Concrete Repair Association, Farmington Hills, MI.

[28] Phillips, J. (2011) A Modeling and Simulation Framework for Electrokinetic Nanoparticle Treatment. Louisiana Tech University, Ruston, Louisiana.

[29] Harris, D.C. (2012) Exploring Chemical Analysis. Macmillan, New York City. 